\title{
Lipreading Ability and Its Cognitive Correlates in Typically Developing Children and Children With Specific Language Impairment
}

\section{Heikkilä, Jenni}

2017-03

Heikkilä , J , Lonka , E , Ahola , S , Meronen , A \& Tiippana , K 2017 , ' Lipreading Ability and Its Cognitive Correlates in Typically Developing Children and Children With Specific

Language Impairment ' , Journal of Speech, Language, and Hearing Research, vol. 60 , no.

3 , pp. 485-493 . https://doi.org/10.1044/2016_JSLHR-S-15-0071

http://hdl.handle.net/10138/310050

https://doi.org/10.1044/2016_JSLHR-S-15-0071

acceptedVersion

Downloaded from Helda, University of Helsinki institutional repository.

This is an electronic reprint of the original article.

This reprint may differ from the original in pagination and typographic detail.

Please cite the original version. 
Lipreading ability and its cognitive correlates in typically developing children and children with specific language impairment (SLI)

Jenni Heikkilä ${ }^{1}$, Eila Lonka ${ }^{2}$, Sanna Ahola ${ }^{1}$, Auli Meronen ${ }^{3}$, Kaisa Tiippana $^{1}$

${ }^{1}$ Division of Cognitive Psychology and Neuropsychology, Institute of Behavioural Sciences, University of Helsinki \& ${ }^{2}$ Division of Logopedics, Institute of Behavioural Sciences, University of Helsinki, Finland. ${ }^{3}$ Niilo Mäki Institute, Jyväskylä, Finland.

Correspondence concerning this article should be addressed to:

Jenni Heikkilä

Division of Cognitive Psychology and Neuropsychology, Institute of Behavioural Sciences, University of Helsinki.

P.O. Box 9, 00014 University of Helsinki, Finland.

E-mail: jenni.heikkila@helsinki.fi; Tel: +358 503199448 


\begin{abstract}
Purpose: Lipreading and its cognitive correlates were studied in school-aged children with typical language development and delayed language development due to specific language impairment (SLI).
\end{abstract}

Method: Forty-two children with typical language development and twenty children with SLI were tested using a word-level lipreading test and an extensive battery of standardized cognitive and linguistic tests.

Results: Children with SLI were poorer lipreaders than their typically developing peers. Good phonological skills were associated with skilled lipreading in both typically developing children and in children with SLI. Lipreading was also found to correlate with several cognitive skills, for example short-term memory capacity and verbal motor skills.

Conclusion: Speech processing deficits in SLI extend also to the perception of visual speech. Lipreading performance was associated with phonological skills. Poor lipreading in children with SLI may be thus related to problems in phonological processing.

Keywords: Lipreading; Children; Specific language impairment (SLI); speech 


\section{Introduction}

Visual information from a speaker's face plays an important role in understanding spoken language. In noisy conditions, watching speaker's facial movements improves speech perception (Sumby \& Pollack, 1954; MacLeod \& Summerfield, 1987). This improvement can be observed even when the auditory signal is intact (Reisberg, Mclean \& Goldfield, 1987). Children are sensitive to visual speech from infancy, and perception of visual speech can aid the development of language (Lewkowicz \& Hansen-Tift, 2012; see Soto-Faraco, Calabresi, Navarra, Werker \& Lewkowicz, 2012 for review). It is also possible to understand spoken language by only observing the facial gestures of a speaker, without any auditory cues. The term lipreading refers to this ability to perceive visual speech alone (Bernstein \& Liebenthal, 2014). It is most commonly studied in connection with hearing impairment, but in this paper we shall concentrate on the lipreading skills of people with normal hearing. The aim of this study was to investigate lipreading skills and its cognitive correlates in school-aged children with typical language development and delayed language development due to specific language impairment (SLI).

\section{Lipreading skills in typical and delayed language development}

Adults are able to lipread, at least to some extent (Bernstein, Demorest \& Tucker, 2000; Ellis, Macsweeney, Dodd \& Campbell, 2001; Mohammed, Campbell, Macsweeney, Barry \&Coleman, 2006; Auer \& Bernstein, 2007), and they perform better than children in lipreading tasks (Massaro, Thompson, Barron \& Laren, 1986; Wightman, Kistler \& Brungart, 2006; Sekiyama \& Burnham, 2008; Erdener \& Burnham, 2013). Lipreading ability of typically developing children improves with age (Sekiyama \& Burnham, 2008; Erdener \& Burnham, 2013; Kyle, Campbell, Mohammed, Coleman \& Macsweeney, 2013; Tye-Murray, 
Hale, Spehar, Myerson \& Sommers, 2014), yet contrasting evidence has also been found (Ross et al., 2011; Foxe et al., 2013). Most studies on lipreading concern typically developing participants and participants with hearing impairment. Less is known about the lipreading skills in clinical groups with developmental language disorders. Dyslexic adults (Mohammed et al., 2006) and children (de Gelder \& Vroomen, 1998; Hayes et al., 2003; Ramirez \& Mann, 2005) are poorer lipreaders than individuals without dyslexia.

Recently, an interest has arisen to investigate the contribution of visual speech on how children with developmental language difficulties, for example specific language impairment (SLI), perceive speech. SLI refers to the condition in which a child fails to develop his or her native language while the non-verbal and social skills are within a normal range (Tomblin, Records \& Zhang, 1996; Tomblin, Records \& Buckwater, 1997; Norbury, Thomplin \& Bishop, 1998). In SLI, usually both expressive and receptive language skills are delayed. Problems in phonological processing are often present and the children with SLI usually have difficulties in understanding spoken language. Previously, only two studies investigating audiovisual speech perception in children with developmental language difficulties have also assessed lipreading skills (Meronen, Tiippana, Westerholm \& Ahonen, 2013; Leybaert et al., 2014). Both studies found that children with language difficulties utilize visual speech less in audiovisual conditions, and that they are poorer at lipreading consonants than typically developing children. Meronen et al. (2013) showed that recognition accuracy of three visual consonants was poorer in children with developmental language disorder compared to typically developing children. Leybaert et al. (2014) tested the identification accuracy of six visual consonants in children with SLI. The children were presented visual vowel-consonantvowel syllables, for example [apa], and child's task was to identify the middle consonant. The identification of the consonants was poorer in children with SLI than their typically developing peers. These findings are important because they reveal that speech processing 
deficits in SLI are beyond the auditory modality. If the ability to use visual speech cues is poor, it may contribute (together with auditory difficulties) to weak speech perception in children with developmental language difficulties.

In the current study we focused on the lipreading skills of children with SLI. Instead of using a simple consonant recognition task, we developed a word-level lipreading test. This is because in everyday situations, word-level speech perception is required. The ability to use visual articulatory cues in natural conversations is important, especially if the auditory signal is not optimal. The poor lipreading ability of words, together with problems in other language skills, may influence speech understanding in face-to-face conversation.

\section{Cognitive correlates of lipreading}

Both adults and children show great individual variability in lipreading (Bernstein, Demorest, \& Tucker, 2000; Lyxell \& Holmberg, 2000; Andersson, Lyxell, Rönnberg \& Spens, 2001; Bernstein, Auer \& Tucker, 2001; Hall, Fussell \& Summerfield, 2005; Auer \& Bernstein, 2007; Feld \& Sommers, 2009; Tye-Murray \& al., 2014). Differences in cognitive and linguistic skills can explain some of the variation in lipreading ability. In adults, working memory (Lyxell \& Rönnberg, 1992; Andersson et al., 2001), inference making ability (Lyxell \& Rönnberg, 1989) and phonological skills (Andersson, Lyxell, Rönnberg \& Spens, 2001; Lyxell, Anderson, Borg \& Ohlson, 2003) correlate positively with skilled lipreading. In typically developing children, working memory (Lyxell \& Holmberg, 2000; Tye-Murray et al., 2014) and receptive vocabulary size (Davies, Kidd and Lander, 2009) have been shown to be predictors of lipreading performance. In addition, phonological skills, as measured by a nonword repetition task, correlate positively with lipreading ability in preschool children (Davies et al., 2009). Cognitive correlates of lipreading in individuals with developmental 
language difficulties have been studied very little. Phonological skills are positively correlated with lipreading performance in adults with dyslexia, but not in adults without dyslexic history (Mohammed et al., 2006). In children with SLI, cognitive correlates of lipreading have not been studied previously.

\section{Aims of this study}

The first aim of this study was to investigate word-level lipreading performance in adults, typically developed children and children with SLI. We expected lipreading skills to be better in adults than in children. We also expected children with SLI to be poorer in lipreading than typically developed children, in agreement with Meronen (2013) and Leyebaert et al. (2014).

The second aim of this study was to investigate the cognitive correlates of lipreading ability in typically developing children and children with SLI by using several standardized linguistic and cognitive measures, including measures of phonological abilities, short-term memory, language comprehension and production, attention and general cognitive abilities. Visual speech signal is poorly specified because some phonetic features and phonetic distinctions do not have articulatory correlates that are visible, and some phonemes share visual articulatory characteristics that make them easy to confuse when the auditory signal is missing (Lidestam \& Beskow, 2006). Because of the ambiguity of visual speech, effective lipreading may require both recognition of visual articulatory movements and ability to convert these movements into phonemes in order to understand the meaning. Rönnberg, Samuleson and Lyxell (1998) proposed that phonological processing is a prerequisite for (sentence level) lipreading. If so, lipreading should depend partly on phonological skills. We hypothesize that phonological ability may be related to lipreading skills also in school-aged children, as has been shown for pre-school children (Davies \& al., 2009). There are no 
previous studies on the cognitive correlates of lipreading in children with SLI. However, we assume that lipreading may be related to same skills in children with SLI as in typically developing children. If lipreading requires phonological skills, then lipreading performance might be poorer in children with SLI than in typically developing children due to the impairment of these skills.

\section{Methods}

\section{Participants}

Eighty-two participants were recruited in this study. They were twenty adults, forty-two typically developing (TD) children (aged between 6;9 and 11;1 years, mean age 8;3) and twenty children with a SLI diagnosis (aged between 7;2 and 10;8 years, mean age 8;9 years). All children with SLI were diagnosed by professionals (medical doctors), and met the national diagnostic criteria for SLI (Käypähoito, 2010). Children with comorbid neurological or psychiatric diagnosis (ADHD, autism spectrum disorders, general cognitive deficit) were excluded. Children with SLI were recruited from a school that is specialized on children with developmental difficulties. All participants had Finnish as their mother tongue, and they reported normal hearing and normal or corrected to normal vision. The research has received ethical approval from the University of Helsinki Review Board in the Humanities and Social and Behavioural Sciences.

\section{Lipreading test}

A computer-based test was developed to measure word-level lipreading performance in Finnish-speaking, school-aged children. The test was similar to the lipreading tests for 
English-speaking children developed by Kyle et al. (2013) and Tye-Murray et al. (2014). In one trial (Fig. 1), a silent video clip was shown where a native Finnish female speaker uttered a word. Four pictures were then presented: one that matched the word, and three distractor pictures. The participant's task was to lipread the word and to select the corresponding picture by pointing it out. The non-verbal response was used because many of the children with SLI had problems in expressive language skills.

The lipreading test included 17 Finnish words which were common, concrete nouns familiar to children. The word length varied between two and four syllables. The words were selected based on both salient lipreading features and a mixture of easy and difficult words. The distractor words were related to the target words phonetically and in terms of lipreading features. They had the same number of syllables as the target words. The pictures used to indicate the response options were 68 colorful drawings from a picture database created and tested by Rossion and Pourtois (2004). The list of words and pictures is presented in Appendix 1. The lipreading test was presented and data collected using Presentation software (Neurobehavioral systems).

All participants were assessed individually in a quiet room. The participant sat in a chair approximately $50 \mathrm{~cm}$ from the laptop monitor, where the stimuli appeared. The size of the face on the screen was $6.5 \mathrm{~cm}$ in height and $4 \mathrm{~cm}$ in width. Before the testing session, oral instructions were given. The experimenter sat next to the participant during the testing in order to ensure that he/she looked at the screen.

Figure 1 here 


\section{Cognitive Tests}

Children's language skills, general cognitive abilities, attentional skills and working memory capacity were assessed using standardized psychological tests in Finnish. Cognitive assessment was conducted after the lipreading test individually for each child.

Phonological skills were assessed using Phonological Processing of NEPSY-II (Korkman, Kirk \& Kemp, 2008) and Repetition of Nonsense Words of NEPSY (Korkman, Kirk \& Kemp, 1997). The Phonological Processing subtest evaluates the ability to perceive word structure. The subtest consists of two tasks designed to assess phonemic awareness. The task Word Segment Recognition requires identification of words from word segments. The task Phonological Segmentation evaluates phonological processing at the level of word segments (syllables) and speech sounds (phonemes). The child is asked to repeat a word and then to create a new word by omitting a syllable or a phoneme, or by substituting one phoneme in a word for another. ). Repetition of Nonsense Words measures the ability to analyze and reproduce phonological knowledge. In the subtest, the child is asked to repeat spoken nonwords of varying length and complexity.

Verbal short-term memory was assessed by Digit Span of WISC-IV (Wechsler, 2010) and Repetition of Sentences of NEPSY (Korkman, Kirk \& Kemp, 1997). In Digit span, a list of numbers is read aloud, and the task is to orally repeat the numbers. In Repetition of Sentences, the child is asked to repeat sentences of varying length and complexity.

Verbal comprehension and auditory short-term memory were assessed by Comprehension of Instructions of NEPSY-II (Korkman, Kirk \& Kemp, 2008). The child has to touch colored figures according to increasingly complex oral instructions. 
Vocabulary and naming abilities were measured with the Boston Naming Test (Kaplan,Goodglass \& Weintraumb, 1983), in which the child is asked to name pictures. Verbal fluency was evaluated using Word Generation of NEPSY-II (Korkman, Kirk \& Kemp, 2008), in which the child is asked to say as many words as possible in a certain semantic (animals/foods) or phonetic category (the first letter is $\mathrm{s} / \mathrm{k}$ ) in one minute.

Verbal motor skills was assessed by subtest Oromotor Sequences of NEPSY (Korkman, Kirk \& Kemp, 1997), which measures verbal motor coordination and oral praxic functions. The child is asked to repeat sequences of words and syllables

Manual sensorimotor skills were assessed by NEPSY-II (Korkman, Kirk \& Kemp, 2008) subtest Imitating Hand Positions. In the subtest, the child imitates various hand positions presented by the examiner.

Attentional skills was evaluated by subtest Visual Attention of NEPSY-II (Korkman, Kirk \& Kemp, 2008), which assesses the ability to focus and maintain attention to a visual target. In the task, the child searches for pictures of two target faces that are embedded among faces differing in various features.

Non-verbal intelligence was evaluated by Raven's Progressive Matrices (Raven, Raven, \& Court, 1998). 


\section{Results}

\section{Performance in the lipreading test}

The adults mastered the lipreading test almost perfectly, with a mean of $93 \%$ correct responses (varying between $71-94 \%$ ). For the group of TD children, the mean percentage of correct responses was 60\% (varying between 12-94\%). For the group of SLI children, the mean percentage of correct responses was 42\% (varying between 18-71\%) - see Figure 2.

Figure 2 here

An analysis of variance with factors Group (adults, TD children, SLI children) revealed a main effect of Group $\left[\mathrm{F}(2,78)=35.7, \mathrm{p}<.001, \eta^{2}=.475\right]$. Bonferroni-corrected pairwise comparisons showed that lipreading scores were significantly better in adults than in either group of children $(\mathrm{p}<.001$ in both comparisons). Furthermore, the two groups of children differed significantly $(\mathrm{p}=.003)$ : the SLI group was significantly poorer in lipreading than the TD group.

\section{Discrimination sensitivity of the lipreading test for TD and SLI groups}

Receiver Operating Characteristic (ROC) curve was used to analyze the discrimination sensitivity of the lipreading test for SLI and TD groups. In clinical studies, ROC curves are 
used to measure the ability of a test to differentiate patients from non-patients (see Zweig \& Campbell, 1993; Akobeng, 2007; Lalkhen \& McCluskey, 2008 for methodological reviews). A ROC curve is a plot of true positive rate (sensitivity) against false positive rate (1specificity) at several potential cutoff scores of the test. The Area Under the Curve (AUC) is a measure of the discriminative potential of a clinical test. It gives the probability that a randomly selected patient will give a lower score than a randomly selected non-patient. Thus, the closer the AUC is to 1.0, the better the test is able to discriminate patients from nonpatients. If the AUC is 0.5 , the test does not discriminate at all. The lipreading test scores of the SLI and TD children were used in the ROC analysis in order to analyze how well the test discriminates children with SLI from typically developing children. That is, the true positive rate (number of children with SLI correctly identified by the lipreading test) and the false positive rate (number of TD children incorrectly identified as having SLI) were plotted at various cutoff points (lipreading scores). The resulting ROC curve gave an AUC of .731, suggesting that the lipreading test has a moderate accuracy in discriminating between TD and SLI groups (Akobeng, 2007). The lipreading test can therefore be used to differentiate between individuals with and without SLI, but it is not as accurate as for example nonword repetition and sentence repetition tests, which have been studied previously (Conti-Ramsden, Botting \& Faragher, 2001).

\section{Comparison of the cognitive skills of children with SLI and TD children}

The cognitive test performance of children with SLI was significantly poorer compared to their typically developing peers in subtests Comprehension of Instructions $[\mathrm{t}(59)=7.82$, $\mathrm{p}<.001]$, Phonological Processing [t(59)=3.43, $\mathrm{p}=.02]$, Word Generation $[\mathrm{t}(59)=4.10$, $\mathrm{p}<.001]$, Repetition of Sentences [t(59)=9,46, $\mathrm{p}<.001]$, Repetition of Nonsense Words 
$[\mathrm{t}(59)=7.15, \mathrm{p}<.001]$, Boston Naming Test $[\mathrm{t}(59)=5,10, \mathrm{p}<.001]$, Digit Span $[\mathrm{t}(59)=5,88$, $\mathrm{p}<.001]$, Oromotor Sequences $[\mathrm{t}(59)=5,74, \mathrm{p}<.001]$ and Imitating Hand Positions $[\mathrm{t}(59)=4.75, \mathrm{p}<.001]$. The groups did not differ in subtests Raven Matrices $[\mathrm{t}(59)=2.12$, $\mathrm{p}>.05]$ and Visual Attention $[\mathrm{t}(59)=2.72, \mathrm{p}<.05]$. The means and ranges for all cognitive tests are presented in Table 1.

Table 1 here

\section{Relationship between group, lipreading and cognitive skills}

In order to study whether the pattern of correlations between the lipreading test and any cognitive test differed for the two groups of children (TD, SLI), we conducted regression analyses to examine the relationship of cognitive test performance and group on lipreading performance. There were no group differences in any cognitive test in relation to lipreading ( $p>0.5$ in each analysis). Because there were no group differences, the data of TD and SLI groups were merged for further analyses $(n=62)$.

The relationship between lipreading and cognitive test scores was examined with Pearson's product moment correlations. There were significant positive correlations between lipreading and Repetition of Nonsense Words, Phonological Processing, Repetition of Sentences, Comprehension of Instructions, Digit Span, Oromotor Sequences, Boston Naming Test, Raven Matrices and Imitating Hand Positions (Table 2).

To investigate these relationships further, a regression analysis was carried out to determine the factors associated with the lipreading ability. Estimations were carried out using stepwise 
regression analysis. The dependent variable was the lipreading test score. The selection of the independent variables was based on the correlation analysis.

Table 2 here

Repetition of Nonsense Words was the strongest correlate and it was entered to the model first, followed by Phonological Processing, Repetition of Sentences, Comprehension of Instructions, Digit Span and Oromotor Sequences. Best explanatory power was found with factors Repetition of Nonsense Words and Phonological Processing, accounting 33\% of the variance in lipreading ability (Table 3 ). The model had statistically significant explanatory power $\left(F(2,58)=15.79, p<0.001\right.$, Adjusted $\left.R^{2}=0.33\right)$. Adding the remaining variables did not account any additional variance of the lipreading score (Table 4 ).

Table 3 here

\section{Discussion}

The aim of this study was to investigate lipreading skills in adults and children, and to study which cognitive skills are associated with lipreading in children. We investigated this by assessing performance in a lipreading test, as well as in several cognitive and linguistic tests in children with typical language development and in a children with SLI. 
Adults outperformed both groups of children in the lipreading test, in line with previous studies (Massaro et al., 1986; Wightman et al., 2006; Sekiyama \& Burnham, 2008; Erdener \& Burnham, 2013). The group of typically developing children had higher lipreading scores than the group of children with SLI. This finding provides further evidence that lipreading is impaired in children with SLI by showing that not only lipreading of consonants (Meronen et al., 2013; Leybaert et al., 2014) but also lipreading of meaningful words is impaired in SLI. Children with SLI thus do not understand visual speech as well as typically developing children. Problems in word-level lipreading skills may affect speech understanding in face-toface conversations, especially when other language skills are below average.

There was great variation in lipreading skills among children in both groups, and we expected part of this variation may be explained by the cognitive skills required in the lipreading process. There were no differences between the TD and SLI group in any cognitive tests in relation to lipreading.. Good phonological skills (Phonological Awareness and Repetition of Nonsense Words) were associated with skilled lipreading. Previously, phonological skills have been found to correlate with lipreading ability in adults (Andersson et al., 2001; Lyxell et al., 2003) and preschool children (Davies et al., 2009).

In the study of Tye-Murray et al. (2014), performance in their phonological processing task did not predict lipreading. However, they used a task in which a series of separate speech sounds was presented (e.g. $[\mathrm{m}],[\mathrm{u}]$ and $[\mathrm{n}]$ ), and participants were asked to blend the sounds together to make a meaningful word (e.g. moon). This task measures the ability to synthesize speech sounds to form words. In contrast, our phonological tasks measure the ability to understand and manipulate the phonemic and syllabic structure of words. The task differences may explain the different results found in these studies. Lipreading may require more complex phonological abilities than forming words from separate sounds. 
Lipreading differs from understanding auditory speech because all phonemes are not visible on the face and therefore visual speech does not provide as much phonetic information as auditory speech (Lidestam \& Beskow, 2006). Therefore, a lipreader can perceive only parts of the word, and the rest of the information must be inferred. Understanding the phonetic structure of the language and how phonemes are used to build up meaningful words are a prerequisite for good lipreading skills, according to Rönnberg et al. (1998). Good phonological skills may help children to recognize the articulatory gestures of the visual speech and parse them into a meaningful word. In children with SLI, phonological skills are weaker than in children with typical language development (Conti-Ramsden et al., 2001; Bishop \& Snowling, 2004). Problems in phonological skills may contribute to the poorer lipreading performance observed in children with SLI. However, there may be other skills than those tested in this study that can account for the lipreading performance of children with SLI.

Short-term memory capacity (Digit Span, Repetition of Sentences, Comprehension of Instructions) correlated positively with lipreading. Previous studies have shown that working memory predicts lipreading in children (Lyxell \& Holmberg, 2000, Tye-Murray et al., 2014). Together, these findings suggest that memory capacity plays a role in lipreading. Short-term memory may be important because low memory capacity may offer less ability to encode and store visual speech cues. Individuals with low short-term memory capacity have problems in remembering auditory speech, and the same problems may be present in visual speech processing.

Deficits in speech production may also contribute to problems in lipreading, as verbal motor skills correlated with lipreading scores. Children who had problems in speech production and articulation also had problems in lipreading. This implies that a child's ability to guide her/his own articulatory gestures may affect the way she/he can perceive the articulatory gestures of 
other people. Tye-Murray et al. (2015) showed that participants obtained greater benefits from visual speech in audiovisual speech perception, when they saw their own speech compared to speech of other talkers. This suggest that there is a strong link between motor and sensory speech representations, and this linkage is important also in visual speech recognition. Desjardins, Rogers \& Werker (1997) found that preschool children who made more substitution errors in speech production tasks performed worse in a syllable-level lipreading task compared to children who did not make speech errors. They concluded that experience in producing speech plays a role in the elaboration of the visual speech representation. It may be that problems in speech production contribute to the poorer performance in lipreading. Even though short-term memory capacity and verbal motor skills were not associated with lipreading ability in our regression analysis, more research is needed on this topic.

In conclusion, the results of this study show that children with SLI are poorer at lipreading than their typically developing peers. Phonological skills are associated with lipreading ability. We propose that understanding the phonetic structure of the language and how phonemes are used to build up meaningful words are a prerequisite for good lipreading skills. Lipreading may thus be poorer in children with SLI partly because their phonological skills are weaker than in children with typical language development.

\section{Acknowledgements}

This research was funded by grants from the University of Helsinki and Arvo and Lea Ylppö Foundation. We are grateful to the University of Jyväskylä and Valteri Centre for Learning and Consulting, Onerva, Jyväskylä, Finland, and to the pupils and teachers in Valteri School 
Onerva, Jyväskylä, Finland and Töölö Primary School, Helsinki, Finland, where the research was conducted.

\section{References}

Akobeng, A. (2007). Understanding diagnostic tests 3: receiver operating characteristic curves. Acta Paediatrica, 96, 664-7.

Andersson, U., Lyxell, B., Rönnberg, J. \& Spens, K. (2001). Cognitive correlates of visual speech understanding in hearing-impaired individuals. Journal of Deaf Studies and Deaf Education, 6:2, 103-115.

Auer, E. T. Jr. \& Bernstein, L. E. (2007). Enhanced visual speech perception in individuals with early onset hearing impairment. Journal of Speech, Language, and Hearing Research, 50(5), 1157-1165.

Bernstein, L. E., Auer, E. T. Jr., \& Tucker, P. E. (2001). Enhanced speechreading in deaf adults: can short-term training/practice close the gap for hearing adults? Journal of Speech, Language, and Hearing Research, 44(1), 5-18.

Bernstein, L. E., Demorest, M. E. \& Tucker, P. E. (2000). Speech perception without hearing. Perception and Psychophysics, 62(2), 233-252. 
Bernstein, L. E. \& Liebenthal, E. (2014). Neural pathways for visual speech perception. Frontiers in Neuroscience, 8.

Bishop, D. \& Snowling, M. (2004). Developmental dyslexia and specific language impairment: same or different? Psychological bulletin, 130, 858-86.

Conti-Ramdsen, G., Botting, N. \& Faragher, B. (2001). Psycholinguistic markers for Specific Language Impairment (SLI). Journal of Child Psychology and Psychiatry, 42, 741-48

Davies, R., Kidd, E. \& Lander.K. (2009). Investigating the psycholinguistic correlates of speechreading in preschool age children. International Journal of Language and Communication Disorders, 44, 2, 164-74.

de Gelder, B., \& Vroomen, J. (1998). Impaired speech perception in poor readers: evidence from hearing and speech reading. Brain and Language, 64(3), 269-281.

Desjardins, R., Rogers, J. \& Werker, J. (1997). An exploration of why preschoolers perform differently than do adults in audiovisual speech perception tasks. Journal of Experimental Child Psychology, 66, 85-110.

Ellis, T., Macsweeney, M., Dodd, B. \& Campbell, R. (2001). TAS: A new test of adult speechreading. Deaf people really can be better speechreaders. AVSP 2001, International Conference of Audiovisual Speech Processing. 
Erdener, D. \& Burnham, D. (2013). The relationship between auditory-visual speech perception and language-specific speech perception at the onset of reading instruction in English-speaking children. Journal of Experimental Child Psychology, 116, 120-38.

Feld, J. \& Sommers, M. (2009). Lipreading, processing speed, and working memory in younger and older adults. Journal of Speech, Language and Hearing Research, 52,155565.

Foxe, J., Molholm, S., Del Bene, V., Frey, H., Russo, N., Blanco, D., Saint-Amour, D. \& Ross, L. (2013). Severe multisensory speech integration deficits in higher-functioning school-aged children with autism spectrum disorder (ASD) and their resolution during early adolescence. Cerebral Cortex, 25, 298-31

Hall, D. A., Fussell, C. \& Summerfield, A. Q. (2005). Reading fluent speech from talking faces: typical brain networks and individual differences. Journal of Cognitive Neuroscience, 17(6), 939-953.

Hayes, E. A., Tiippana, K., Nicol, T. G., Sams, M., \& Kraus, N. (2003). Integration of heard and seen speech: a factor in learning disabilities in children. Neuroscience Letters, 351(1), 46-50.

Kaplan, E., Goodglass, H. \& Weintraub, S. (1983). Boston Naming Test. Philadelphia: Lea \& Febiger. 
Korkman, M., Kirk, U. \& Kemp, S.L. (1997). NEPSY, Lasten Neuropsykologinen Tutkimus (The assessment of neuropsychological abilities in children). Helsinki: Psykologien Kustannus, 1997.

Korkman, M., Kirk, U. \& Kemp, S.L. (2008). NEPSY-II, Lasten Neuropsykologinen Tutkimus (The assessment of neuropsychological abilities in children). Helsinki: Psykologien Kustannus, 2008.

Kyle, F., Campbell, R., Mohammed,T., Coleman, M. \& Macsweeney, M. (2013). Speechreading development in deaf and hearing children: Introducing the test of child speechreading. Journal of Speech, Language and Hearing Research, 56, 416-26.

Käypähoito recommendations (2010): DOI:

http://www.kaypahoito.fi/web/kh/suositukset/suositus?id=nix01570\&suositusid=hoi50085

Lewkowicz, D.J. \& Hansen-Tift, A. (2012). Infants deploy selective attention to the mouth of a talking face when learning speech. Proceedings of the National Academy of Sciences, $109(5), 1431-36$.

Leybaert, J., Macchi, L., Huyse, A., Champoux, F., Bayard, C., Colin, C. \& Berthommier, F. (2014). Atypical audio-visual speech perception and McGurk effects in children with specific language impairment. Frontiers in Psychology, 5.

Lidestam, B. \& Beskow, J. (2006). Visual phonemic ambiguity and speechreading. Journal of Speech, Language and Hearing Research, 49, 835-47. 
Lyxell, B., Andersson, U., Borg, E. \& Ohlson, I. (2003). Working-memory capacity and phonological processing in deafened adults and individuals with severe hearing impairment. International Journal of Audiology, 42, 86-89.

Lyxell, B. \& Holmberg, I. (2000). Visual speechreading and cognitive performance in hearing-impaired and normal hearing children (11-14 years). British Journal of Educational Psychology, 70, 505-18.

Lyxell, B. \& Rönnberg, J. (1989). Information-processing skill and speechreading. British Journal of Audiology, 23,339-47.

Lyxell, B. \& Rönnberg, J. (1992). The effects of background noise and working memory capacity on speechreading performance. Scandinavian Audiology, 21.

MacLeod, A. \& Summerfield, Q. (1987). Quantifying the contribution of vision to speech perception in noise. British Journal of Audiology, 21, 131-141.

Massaro D. W., Thompson L. A., Barron B. \& Laren E. (1986). Developmental changes in visual and auditory contributions to speech perception. Journal of Experimental Child Psychology, 41, 93-113.

Meronen, A, Tiippana, K, Westerholm, J. \& Ahonen, T. (2013). Audiovisual speech perception in children with developmental language disorder in degraded listening conditions. Journal of Speech, Language and Hearing Research, 56,211-21. 
Mohammed, T., Campbell, R., Macsweeney, M., Barry, F. \& Coleman, M. (2006). Speechreading and its association with reading among deaf, hearing and dyslexic individuals. Clinical Linguistics and Phonetics, 20,621-30.

Norbury, C.F., Thomplin, J.B. \& Bishop, D. (2008). Understanding developmental language disorders, From theory to practice. East Sussex, UK, Psychology Press.

Raven, J., Raven, J. C. \& Court, J.H. (1998). Coloured Progressive Matrices. Pearson, San Antonio, US.

Ramirez, J., \& Mann, V. (2005). Using auditory-visual speech to probe the basis of noiseimpaired consonant-vowel perception in dyslexia and auditory neuropathy. Journal of the Acoustical Society of America, 118(2), 1122-1133.

Reisberg, D., Mclean, J. \& Goldfield, A. (1987). Easy to heard but hard to understand: A lipreading advantage with intact auditory stimuli. In B.Dodd and R. Campbell (eds), Hearing by the Eye: The Psychology of Speechreading. Lawrence Erlbaum, London, UK.

Ross, L., Molholm, S., Blanco, D., Gomez-Ramirez, M., Saint-Amour, D. \& Foxe, J. (2011). The development of multisensory speech perception continues into the late childhood years. European Journal of Neuroscience, 33, 2329-2337.

Rossion, B. \& Pourtois, G. (2004). Revisiting Snodgrass and Vanderwart's object set: The role of surface detail in basic-level object recognition. Perception, 33, 217-236. 
Rönnberg, J., Samuelson, S. \& Lyxell, B. (1998). Conceptual constrains in sentence-based lipreading in the hearing-impaired. In Dodd, B., Campbell, R. \& Burnham, D. (eds), Hearing by the Eye II: Advances in the Psychology of Speechreading and Auditory-visual Speech. Psychology Press, East Sussex, UK.

Sekiyama, K. \& Burnham, D. (2008). Impact of language development of auditory-visual speech perception. Developmental Science, 11(2), 306-20.

Soto-Faraco, S., Calabresi, M., Navarra, J., Werker, J. \& Lewkowicz, D.J. (2012). The development of audiovisual speech perception. In Bremmer, A., Lewkowicz, D.J. \& Spence, C. (eds.). Multisensory Development, Oxford University Press, Oxford, UK.

Sumby W. H. \& Pollack I. (1954). Visual contribution to speech intelligibility in noise. The Journal of the Acoustical Society of America, 26, 212-215.

Tomblin, J., Records, N. \& Buckwater, P. (1997). Prevalence of specific language impairment in kindergarten children. Journal of Speech, Language and Hearing Research, 40, 1245-60.

Tomblin, J., Records, N. \& Zhang, X. (1996). A system for the diagnosis of specific language impairment in kindergarten children. Journal of Speech, Language and Hearing Research, 39, 1284-94.

Tye-Murray, N., Hale, S., Spehar, B., Myerson, J. \& Sommers, M. S. (2014). Lipreading in School-age Children: The Roles of Age, Hearing Status, and Cognitive Ability. Journal of Speech, Language and Hearing Researc, 57, 556-65. 
Tye-Murray, N., Spehar, B., Myerson, J., Hale, S. \&Sommers, M. (2015). The self-advantage in visual speech processing enhances audiovisual speech recognition in noise. Psychonomic Bulletin \& Review, 22, 1048-53

Wechsler, D. (2010). WISC-IV: Wechsler Intelligent Scale for Children (Finnish edition), Helsinki: Psykologien Kustannus, 2010.

Wightman, F., Kistler, D. \& Brungart D. (2006). Informational masking of speech in children: auditory-visual integration. Journal of the Acoustical Society of America, 119, 3940-3949.

Zweig, M. \& Campbell, G. (1993). Receiver-Operating Characteristic (ROC) plots: A fundamental evaluation tool in clinical medicine. Clinical Chemistry, 39, 561-77. 

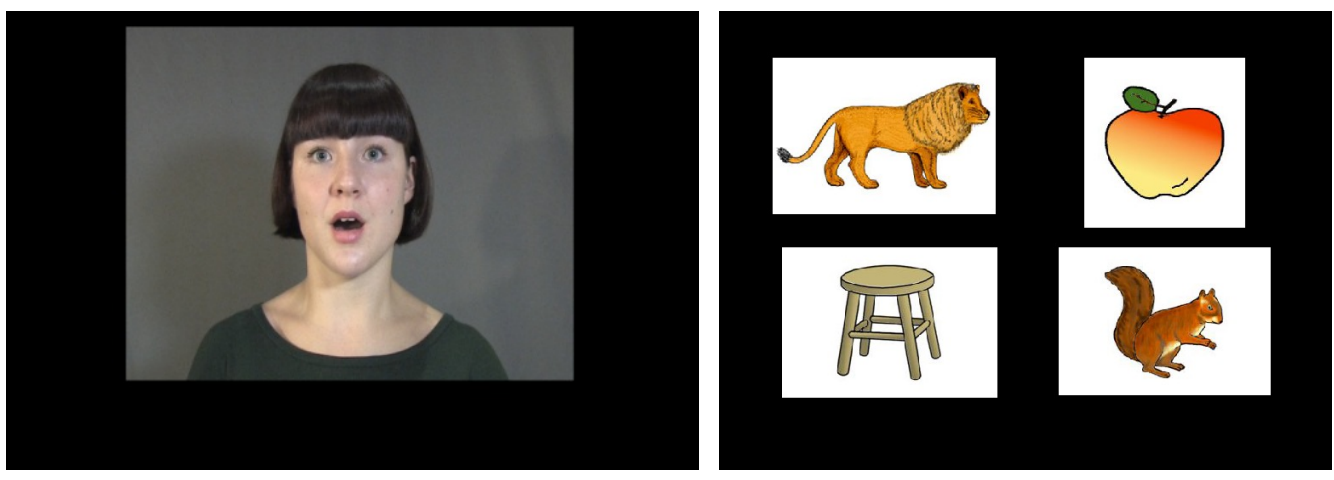

Figure 1: A still of the video clip and an example of pictures in the lipreading test. In the video the speaker is uttering a word ("leijona" = lion). Four drawings are then shown: one that matches the word and three distractors (" omena" = apple, "jakkara" = stool, "orava" = squirrel) in random order on the screen. The task is to lipread the word, and subsequently point at the corresponding picture. 


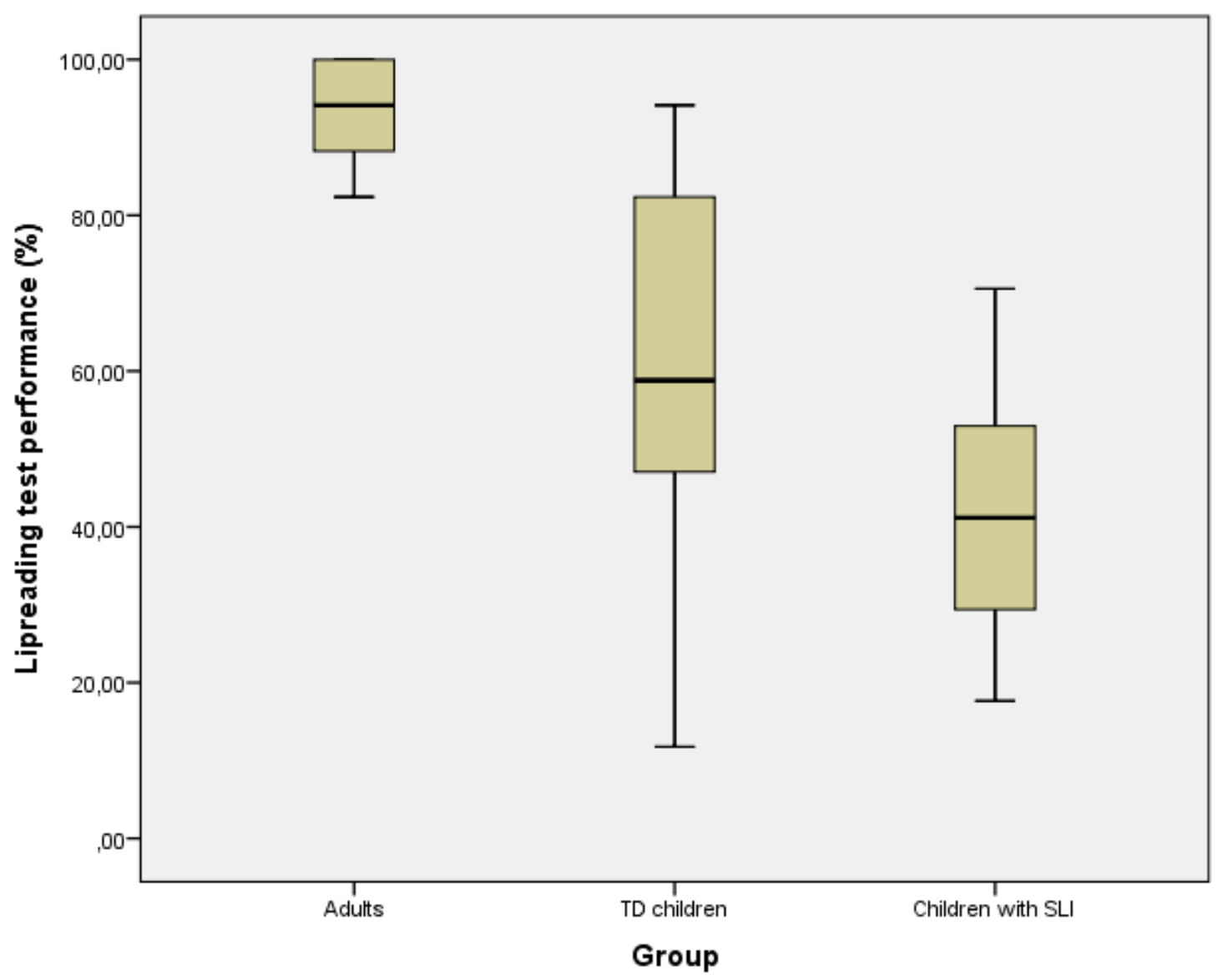

Figure 2. The percentages and ranges of correct responses in the lipreading test for adults, typically developing children and children with SLI. 
Table 1. The mean scores and ranges for cognitive tests (raw scores)

\begin{tabular}{lll}
\hline Test & TD group & SLI group \\
\hline Raven Matrices & $28,3(17-36)$ & $25.1(13-33)$ \\
Digit Span* & $12.0(8-18)$ & $7.7(3-13)$ \\
Boston Naming Test* & $45.4(22-56)$ & $36.6(27-48)$ \\
Phonological Processing* & $38.7(27-50)$ & $31.3(14-48)$ \\
Repetition of Nonsense Words* & $11.5(6-15)$ & $6.1(1-11)$ \\
Comprehension of Instructions* & $26.5(15-32)$ & $20.3(15-26)$ \\
Repetition of Sentences* & $25.1(9-30)$ & $15.3(8-21)$ \\
Word Generation* & $39.1(4-64)$ & $26.0(7-47)$ \\
Oromotor Sequences* & $58.6(18-68)$ & $34.9(0-61)$ \\
Imitating Hand Positions* & $20.6(14-24)$ & $16.4(7-21)$ \\
Visual Attention & $12,2(-36-24)$ & $5.0(-11-19)$ \\
& & \\
\hline
\end{tabular}

* Statistically significant difference $(\mathrm{p}<0.05)$ in test scores between TD group and SLI group. 
Table 2. Correlations between lipreading test scores and cognitive test scores

Cognitive test

Age

Raven Matrices

Digit Span

Boston Naming Test

Phonological Processing

Repetition of Nonsense Words

Comprehension of Instructions

Repetition of Sentences

Word Generation

Oromotor Sequences

Imitating Hand Positions

Visual Attention $\mathrm{r}=.157, \mathrm{p}=.1 .0$

$\mathrm{r}=.330, \mathrm{p}=.012^{*}$

$\mathrm{r}=.481, \mathrm{p}=.001 *$

$\mathrm{r}=.442, \mathrm{p}=.001 *$

$\mathrm{r}=.519, \mathrm{p}=.001^{*}$

$\mathrm{r}=.520, \mathrm{p}=.001^{*}$

$\mathrm{r}=.484, \mathrm{p}=.001$ *

$\mathrm{r}=.508, \mathrm{p}=.001^{*}$

$\mathrm{r}=.-181, \mathrm{p}=1.0$

$\mathrm{r}=.462, \mathrm{p}=.001 *$

$\mathrm{r}=.367, \mathrm{p}=.048^{*}$

$\mathrm{r}=.352, \mathrm{p}=.072$

* Statistically significant correlation $(\mathrm{p}<0.05)$. 
Table 3. Regression analysis of lipreading

\begin{tabular}{cccc}
\hline variable Adj. R2 & $\beta$ & stand. $\beta$ & $\mathrm{p}$ \\
\hline 0.330 & & & \\
Phonological processing & .162 & .347 & .009 \\
Repetition of Nonsense Words & .358 & .324 & .014 \\
\hline
\end{tabular}


Table 4: Change statistics of regression analysis

\begin{tabular}{|l|r|r|r|r|}
\hline & & \multicolumn{3}{|c|}{ Change Statistics } \\
\cline { 3 - 5 } $\begin{array}{l}\text { Variable added to regression } \\
\text { model (in oreder of entry) }\end{array}$ & $\begin{array}{c}\text { Adjusted } \mathrm{R} \\
\text { Square }\end{array}$ & $\begin{array}{c}\text { R Square } \\
\text { Change }\end{array}$ & F Change & $\begin{array}{c}\text { Signifigance of } \\
\text { F Change }\end{array}$ \\
\hline Repetition of Nonsense Words & 0,258 & 0,271 & 21,883 & 0,000 \\
Phonological Processing & 0,330 & 0,082 & 7,341 & 0,009 \\
Sentence repetition & 0,325 & 0,006 & 0,524 & 0,472 \\
Comprehension of Instructions & 0,313 & 0,000 & 0,003 & 0,955 \\
Digit Span & 0,302 & 0,002 & 0,129 & 0,720 \\
Oromotor Sequences & 0,290 & 0,001 & 0,086 & 0,770 \\
\hline
\end{tabular}

